\title{
Understanding the Structure of the Turbulent Mixing Layer in Hydrodynamic Instabilities
}

\author{
D. Laney, P.-T. Bremer, Member, IEEE, A. Mascarenhas, P. Miller, and V. Pascucci, Member, IEEE
}

\begin{abstract}
-
When a heavy fluid is placed above a light fluid, tiny vertical perturbations in the interface create a characteristic structure of rising bubbles and falling spikes known as Rayleigh-Taylor instability. Rayleigh-Taylor instabilities have received much attention over the past half-century because of their importance in understanding many natural and man-made phenomena, ranging from the rate of formation of heavy elements in supernovae to the design of capsules for Inertial Confinement Fusion.

We present a new approach to analyze Rayleigh-Taylor instabilities in which we extract a hierarchical segmentation of the mixing envelope surface to identify bubbles and analyze analogous segmentations of fields on the original interface plane. We compute meaningful statistical information that reveals the evolution of topological features and corroborates the observations made by scientists. We also use geometric tracking to follow the evolution of single bubbles and highlight merge/split events leading to the formation of the large and complex structures characteristic of the later stages. In particular we (i) Provide a formal definition of a bubble; (ii) Segment the envelope surface to identify bubbles; (iii) Provide a multi-scale analysis technique to produce statistical measures of bubble growth; (iv) Correlate bubble measurements with analysis of fields on the interface plane; (v) Track the evolution of individual bubbles over time. Our approach is based on the rigorous mathematical foundations of Morse theory and can be applied to a more general class of applications.
\end{abstract}

Index Terms - topology, multi-resolution, Morse theory

\section{Introduction}

I.3.8 Computer Graphics Applications J.2 [Physical Sciences and Engineering]: Physics;

Understanding the turbulent mixing of fluids is one of the fundamental research problems in the area of fluid dynamics. Turbulent mixing occurs in a broad spectrum of phenomena ranging from boiling water to astrophysics and nuclear fusion. In the present work we apply topological techniques to the analysis of Rayleigh-Taylor instabilities.

Rayleigh-Taylor instability (RTI) occurs when two fluids of different density are accelerated opposite the mean density gradient. That is, a heavier fluid is accelerated against a lighter fluid. In this paper, the RTI occurs under the force of gravity, when a heavy fluid is placed above a light fluid and the plane initially separating them (hereafter called the midplane) is seeded with perturbations. Figure 1 shows the midplane and the progression of the mixing process for one of the simulations analyzed in this paper. The heavy fluid accelerates downward, forming "spikes," while the light fluid moves upward forming "bubbles." The bubbles and spikes are thought to be one way to characterize the large-scale behavior of the mixing process. At the same time, all bubbles and spikes are the result of fluid passing through the midplane, so understanding the properties of fields on the midplane is also an important goal.

Scientists analyzing these simulations are particularly interested in the number of bubbles (and spikes) and their respective evolution. Large-scale models have been proposed based on bubble dynamics in which bubble growth, movement, and interaction are modeled [1]. It is important to understand whether bubbles grow by merging with their neighbors or whether certain bubbles survive and continue to grow while others die-off. A key problem is to create a precise definition of a bubble given the multiscale nature of the fluid flow. Cook et. al. [7] observe four phases in the mixing process resulting from the interaction of entrainment (the influx of unmixed fluid into the mixing

- The authors are with Lawrence Livermore National Laboratory. Corresponding author: Daniel Laney, dlaney@llnl.gov

Manuscript received 31 March 2006; accepted 1 August 2006; posted online 6 November 2006.

For information on obtaining reprints of this article, please send e-mail to: tvcg@computer.org. region) and diffusion:

I Independent growth: The initial perturbations grow independently and no coupling occurs between them.

II Weak turbulence: Coupling between modes occurs as instabilities develop, causing the fluid to begin taking on distinctive mushroom-cap (early bubble) shapes.

III Mixing transition: The mixing rate dominates the entrainment rate, slowing the growth of the mixing layer.

IV Strong turbulence: Mixing and entrainment become balanced, resulting in faster growth of the mixing layer.

These mixing phases affect the shape and number of bubbles. At sufficiently late times the width of the mixing layer has been assumed to be proportional to $t^{2}$, where $t$ is time $[8,6]$. Thus, we expect that a study of bubble dynamics and bubble counts will show the effect of the different observed phases in the mixing process.

Analysis of the simulation data poses challenges at several levels:

1. Theoretical definitions: There exist no prevalent technical definitions of the "features," e.g. bubbles. How can we develop a theoretically sound definition of bubbles amenable to analysis by efficient and robust algorithms?

2. Time-tracking: How do we track bubbles over time to study their birth, merges, splits, or death?

3. Complexity: The late stage mixing creates extremely complex structures and degenerate configurations. How do we avoid numerical problems and exhaustive case-by-case study of degeneracies in the analysis of these structures?

4. Scale: The features of interest can change size drastically over time and must be analyzed independent of scale.

5. Data size: The simulations use high resolution regular grids $\left(1152^{3}\right.$ and $3072^{3}$ in our case) over a large number of time steps (here 755 and 224 respectively). Such large-sized data can render in-core implementations infeasible.

This paper makes contributions to address each of these challenges. Our analysis is performed on two kinds of surfaces: envelope surfaces describing the boundary between undisturbed and "mixed" fluids, and height functions defined on the midplane. 


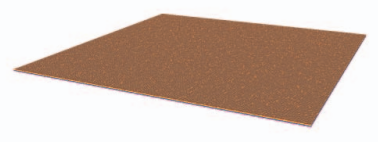

$t=0$

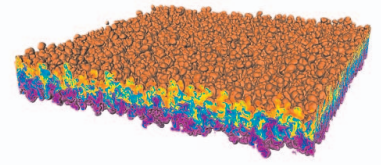

$t=300$

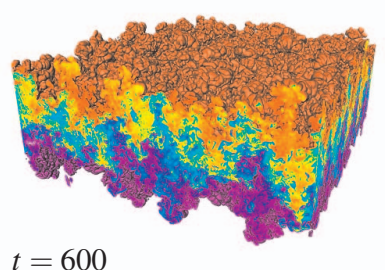

$t=600$

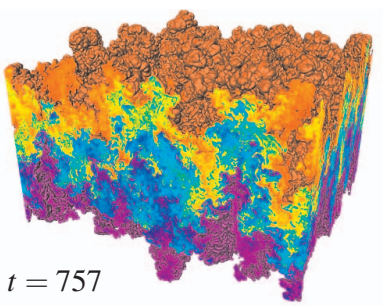

Fig. 1. An overview of a simulation (periodic in $x$ and $y$ ) of Rayleigh-Taylor instability at four time steps on a $1152^{3}$ grid. The light fluid has a density of 1.0, the heavy fluid has a density of 3.0. Two envelope surfaces (at densities 1.02 and 2.98) capture the mixing region. The boundaries of the box show the density field in pseudocolor. The heavy fluid is red and the light fluid is blue. Other colors represent intermediate compositions of mixed fluid. We analyze the upper envelope (red) to study bubble structures and the midplanes to study mixing trends.

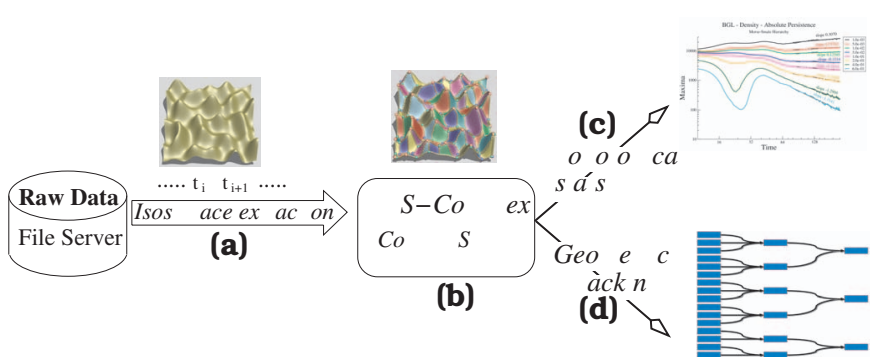

Fig. 2. Processing pipeline for topological data analysis: (a) Extract one isosurface or midplane per time-step; (b) Compute and simplify the MS complex at time $t_{i}$; (c) Store relevant statistical data, e.g. number of critical points; (d) Perform geometric tracking and build merge-split graph.

For both types of surfaces, our definitions and algorithms are based on the mathematical foundations of Morse theory [21] which we present in Section 4. For envelope surfaces, we define bubbles using the constructs of the hierarchical Morse-Smale(MS) complex, implement a robust combinatorial algorithm to construct this complex, and use topological persistence to automatically clean-up noise and identify bubbles at any user-defined scale. We track bubbles over time using a geometric approach to connect the critical points of successive isosurfaces. Section 5 describes the algorithms used to extract and track bubbles and the different types of topological information gathered. We emphasize that all our algorithms are combinatorial in nature and are therefore robust in the presence of noise and degeneracies. The hierarchical nature of our constructions enable us to analyze the data at several scales in both space and time.

For analyzing midplanes, we take advantage of the hierarchical Morse-Smale complex in order to provide persistence-based metrics. We note that Cook et. al. [7] propose a model for the rate of growth of the mixing layer based on the net mass flux through the midplane. Their approach relates the growth rate, in the absence of diffusive effects, to a correlation between density and the Z-component of the velocity at the midplane. In this paper, we analyze the topology of the density and velocity fields on the midplane in order to determine if the mixing phases are discernible and to examine asymptotic behavior in late time. Investigating the topology of these fields constitutes a novel approach.

\section{Overview of our approach}

The different stages of the analysis are pipelined as shown in Figure 2, allowing us to stream through the original data and avoid storing intermediate results. In stage (a), we extract one isosurface per time-step of the data. This sequence of isosurfaces is then fed into stage (b) where we construct the hierarchical MS-complex for each time-step. The hierarchy information from this stage is used in stages (c) and (d) which can operate in parallel. In stage (c), we compute and store the required topological statistics, e.g., bubble counts. In stage (d), we use the hierarchy computed in stage (b) to perform geometric tracking of bubbles over time to produce a graph that indicates how bubbles evolve by merging and splitting. Only the abstract information resulting from the topological analysis is ultimately stored. For example, when generating bubble counts we store the number of bubbles at all topological scales (see Section 4) for each time step. This information is several orders of magnitude smaller than the original raw data and can easily be queried and processed further on an off-the-shelf PC.

During the course of this work we spent several hours with fluid dynamics researchers interactively visualizing the topological features of the envelope surfaces. To accomplish this we computed the isosurfaces - stage (a) - as a preprocess. For small subsets of the data (e.g. $256^{3}$ ) an interactive version of the topological analysis tool allowed us to investigate the topological structures of these surfaces. In order to interact with the MS-complex of the envelope surface at full resolution we also precomputed the MS-complex and stored a compact binary representation of the complex to disk. We modified the streaming mesh viewer of Isenburg et. al. [18] such that a full resolution envelope surface could be simplified and viewed along with its Morse-Smale complex. We describe the specifics of this process in Section 5 .

In this way, visualization was used throughout the project to build up intuition about the mixing behavior of the fluids, and to determine an appropriate model for bubbles (defined in Section 5). Visualization was also used to determine the persistence threshold that segmented the envelope surfaces into bubbles as described in Section 5. For the final data analysis, the work was parallelized over time steps on a Linux cluster, with each node receiving a subset of the time steps to process. In this case, the entire pipeline was executed so that no intermediate isosurface files needed to be stored to disk.

\section{Contributions. We develop:}

1. A formal mathematical definition of a bubble based on the construct of a Morse-Smale complex;

2. A hierarchical segmentation of the mixing envelope surface into bubbles. We analyze the surface at several scales based on persistence and produce statistical measures that corroborate previous observations and independent analysis [7].

3. A method to identify and track individual bubbles over time to study their merge/split/birth/death behavior.

\section{Prior Work}

While models of RT mixing based on bubble interactions have been proposed [1], the direct analysis of bubbles has only recently become a research focus. Kartoon et. al. [9] segment bubbles from a set of small simulations using a vertical velocity criterion. Their method is not fully described in their paper, making it difficult to assess its robustness. They focus on early time dynamics and appear to use a single resolution strategy. Thus, it is likely their method would require significant modification to handle the multiscale late-time behavior observed in the present work.

Topology based methods have become increasingly popular in the area of visualization. They are used directly as a means to provide an abstract visualization as well as to guide traditional visualization 


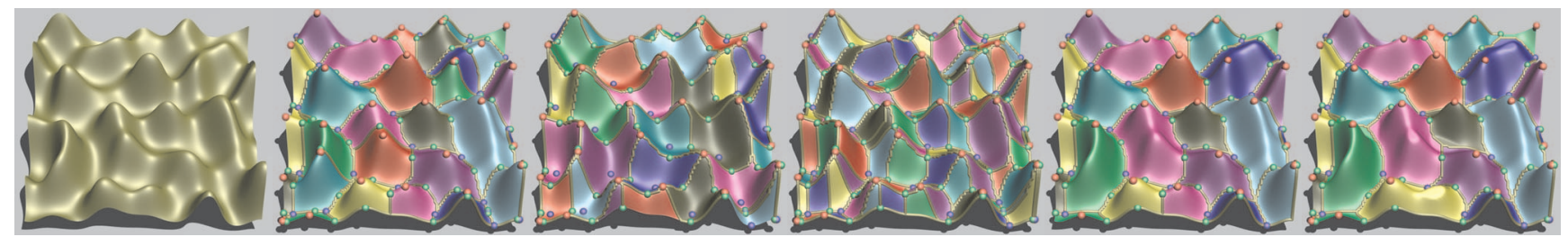

(a)

(b)

(c)

(d)

(e)

(f)

Fig. 3. MS-complex construction, simplification and topologically valid approximation: (a) Small portion of the mixing layer of a Rayleigh-Taylor instability at an early time step; (b) Stable manifolds; (c) Unstable manifolds; (d) MS-complex; (e) Stable manifolds of a simplified complex; (f) Approximation of $f$ corresponding to the topology of (e). Maxima are drawn red, minima blue, and saddles green.

techniques such as isosurfacing or volume rendering. In [14], Edelsbrunner et al. describe the first algorithm to compute the Morse-Smale complex of a function on a 2-manifold, and an algorithm to simplify the function using the notion of topological persistence. In [3] Bremer et al. improve the initial algorithm and propose a hierarchical structure making the algorithm easier to apply and the simplification more flexible. Extensions to 3D can be found in [13] and [16]. Examples of techniques to guide visualization algorithms are the flexible isosurfaces of Carr et al. [5] and automatic design of transfer functions by Weber et al. [32].

Similar work exists for vector field visualization based on the seminal paper of Helman and Hesselink [17]. An overview can be found in [24] and some recent work in [28]. There also exist methods to simplify vector field topology in 2D [10, 30] and three-dimensions [33].

The second aspect of the research presented here is the tracking of features in isosurfaces over time. Samtaney et al. [23] describe a general feature tracking algorithm based on extracting features at each time step, computing descriptive attributes, e.g. center of gravity, size, etc., and solving a best matching problem to create time correspondences. The results are presented as a graph encoding the birth, death, merging, and splitting of features. In some follow-up work they also use the volume overlap of features to decide correspondences [25, 26]. Reinders et al. [22] use very similar ideas combined with motion prediction to improve matching accuracy.

Edelsbrunner et al. [12] develop theory and algorithms to compute time-varying Reeb graphs by connecting them using Jacobi sets [11] which are the paths traced by critical points. Ji et al. $[19,20]$ track the evolution of isosurfaces in a time-dependent volume by extracting (2D) isosurfaces within each time slice and define two such surfaces as linked if they are part of the same three dimensional space-time isosurface. Sohn and Bajaj [2] compute correspondences between contour trees at successive time-steps by using volume matching as in [25] rather than the topological analysis used in [12] and [27]. They use this correspondence to encode the evolution of the isosurface of a fixed isovalue over time in the Topology Change Graph.

Tracking of critical points and/or features is also common in flow field analysis typically based on integrating streamlines/surfaces through space-time. Work on 2D flow fields can be found in [31] and [29] and three dimensional extensions in [15] and [33].

\section{Background: Morse theory}

This section reviews the formal definition of the Morse-Smale complex $[21,3]$ and algorithms to compute and simplify it.

\subsection{Morse-Smale complex}

Given a smooth 2-manifold $\mathbb{M}$ and a smooth function $f: \mathbb{M} \rightarrow \mathbb{R}$, a point $x \in \mathbb{M}$ is critical when its gradient $\nabla f(x)$ vanishes; it is regular otherwise. A critical point $x$ is non-degenerate if the Hessian of $f$ at $x$ has full rank. Finally, a function is Morse if all critical points are non-degenerate and have distinct function values.

An integral line is a maximal path on $\mathbb{M}$ whose tangent vectors agree with the gradient of $f$. The integral lines define a natural seg- mentation of $\mathbb{M}$ because each point in $\mathbb{M}$ has a single integral line passing through it. The stable manifold $S(x)$ of a critical point $x$ is the union of $x$ and all integral lines that end at $x$. The unstable manifold $U(x)$ of $x$ is defined symmetrically as the union of a critical point $x$ and all integral lines that start at $x$. Assuming that no integral line both starts and ends at a saddle one can superimpose the stable and unstable manifolds of all critical points to create the Morse-Smale complex (or MS complex) of $f[14,3]$, see Figure 3(a)-(d). The nodes of this complex are the critical points of $f$, its $\operatorname{arcs}$ are integral lines starting or ending at saddles and its regions are the non-empty intersections of stable and unstable 2-manifolds. By definition, all integral lines within a region start at the same minimum and end at the same maximum and $f$ is monotone. Therefore, the MS complex of $f$ completely describes the topology of $f$. More details on the definition of the MS-complex on 2-manifold triangle meshes and algorithms to compute it are given by Bremer et al. [3].

Simplification. It is often useful to simplify an MS-complex in order to remove noise as well as to analyze functions on multiple scales. Following [3] we use cancellations of connected critical point pairs to simplify an MS-complex. The two possible cases are a maximumsaddle and a minimum-saddle cancellation, examples of the former are shown in Figure 4.

Cancellations are ranked by their persistence - the absolute difference in function value between the two critical points they remove. It can be shown that greedily canceling the the pair with smallest persistence simplifies the topology of $f$ in an $L_{\infty}$-optimal manner. Figures 3(e) and (f) shows an example of a topological simplification and a corresponding approximation of $f$.
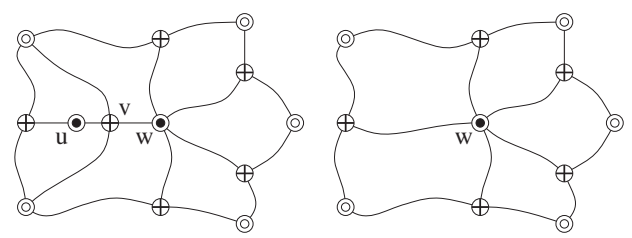

() minimum

- maximum

$\oplus$ saddle

Fig. 4. Morse-Smale complex before and after canceling $u, v$. One saddle, one extremum, four arcs, and two regions are removed.

Computation. In practice, one usually deals with piece-wise linear (PL)-functions given at the vertices of a triangulation. A detailed discussion on how the smooth theory discussed above is transfered to PL-functions can be found in [3]. In particular, we use a slightly modified version of the algorithms by Bremer and Pascucci [4] to efficiently compute and simplify MS-complexes. Starting from saddles, the arcs of the MS-complex are computed as steepest monotone lines that do not cross each other. We avoid all mesh refinement by directly dealing with merged lines as well as multi-saddles. As a result we can use efficient static data structures to store the triangulation allowing us to compute MS-complexes of large data sets common in simulation. 


\section{Analysis}

The focus of our research is to provide a set of dependable tools that scientists can use to analyze mixing behavior. We cannot assume that there exists a particular objective other than to "better understand" the physics involved. Traditionally, application scientists provide some definition of interesting features (a tumor, a vortex core, etc.) and visualization aims to extract and highlight the particular object of interest. In the case of Rayleigh-Taylor simulations however, few concrete objectives exist besides the intuitive notion that the behavior of bubbles and spikes is important. Therefore, our goal is no longer primarily to provide a visualization of the data but to support the data analysis process in general.

An important consequence of analyzing little known phenomena is that results are difficult to validate. Therefore, techniques which cannot be independently proved to work as expected are of little use as they cannot necessarily be trusted. This section describes the various techniques we use to analyze different aspects of the data.

Data. We analyze data from two simulations. Both data sets are defined on regular grids that are periodic in the $X$ and $Y$ dimensions and closed in the $Z$ dimension. The first data set, denoted as Borg, contains data defined on an $1152^{3}$ grid at 759 distinct times. It utilizes a sub-grid scale model for diffusivity and viscosity. The larger simulation, denoted as BGL since it was computed on the BlueGene/L supercomputer, is defined on a $3072^{3}$ grid at 223 time steps and directly models all relevant physical quantities with no subgrid-scale approximations. For each grid point the data contains 4-byte floating point values for density, pressure, and the three velocity components.

Segmentation of bubbles. One of the challenges in analyzing the mixing behavior is that there exists no prevalent mathematical definition for what constitutes a bubble/spike. In general, a bubble can be understood as a three dimensional feature composed of lighter density fluid moving upwards (in the $Z$-direction) into a heavier density fluid. Scalable 3-D Morse-Smale analysis is not yet available, so in consultation with physicists we have determined that segmenting bubbles based on the mixing envelope surface is a reasonable first step. We use the topological concepts introduced in Section 4 to define bubbles, spikes and other features of interest. Consider the images of the segmented mixing envelope surface at different times shown to the left, bottom, and right of the plot in Figure 7. During early time steps (7 upper left and middle left) it is natural to consider the mixing envelope as a time-varying functional surface defined over the $X Y$-plane and associate local maxima with bubbles. This analogy fails at later time steps because the surface becomes non-functional. However, we can generalize this approach by treating the envelope surface as the domain of a function whose value at a point $x$ is the $Z$-coordinate of $x$. It is natural to connect the maxima of this function to bubbles and compute the stable manifold of each maximum as a segmentation of the surface into bubbles. As can be seen in Figure 7, this segmentation corresponds very well to the human notion of a bubble. Symmetrically, we use the unstable manifolds of minima to define spikes. Potentially, other functions could be defined on the envelope surface that would result in a robust segmentation as well. For example, the $Z$-velocity at all of the points on the envelope surface could be incorporated to capture the fact that bubbles should be moving upwards into the heavy fluid. Given the complexity of the problem, it has been determined to use the simplest most intuitive segmentation.

In general, topological based segmentations are often linked to important features: Maximal and minimal $Z$-velocities on the midplanes correspond to cores of rising and falling sections of fluids; Density extrema correspond to pockets of unmixed fluids. This makes topological methods flexible and allows us to analyze a variety of phenomena using the same methodology. Furthermore, the MS-complex can be computed combinatorially $[14,3]$ which translates into provably correct and stable algorithms which are crucial when dealing with large and complex data.

Multi-Scale analysis and persistence selection. The MScomplex, just as any other segmentation, captures noise as well as features. A simplification scheme can be used to remove noise and construct a series of approximations at decreasing resolution. Unlike many other techniques, topological segmentations allow a simplification scheme that is optimal in the $L_{\infty}$-norm. One can formulate the problem of coarsening a segmentation in the following manner: Given a function $f$ and a segmentation $S$ of the domain of $f$, what is the minimal change on $f$ such that $S$ is coarsened? If the segmentation one considers is the MS-complex of $f$, then it can be shown [3] that canceling a critical point pair with persistence $p$ in $f$ requires an approximation $\hat{f}$ with $\|f-\hat{f}\|_{\infty} \geq p / 2$. Therefore, canceling critical points in order of increasing persistence corresponds to an $L_{\infty}$-optimal simplification.

For each MS-complex we compute a sequence of cancellations which optimally simplifies the complex down to its minimal configuration. We can thus define a family of segmentations of the envelope surface ranging from persistence $p=0.0$, where both signal and noise features are segmented, to persistence $p=1.0$ (full function range), where the entire surface is collapsed into a single component. We can then create statistics showing the number of bubbles over time using a range of persistence thresholds. As shown in Section 6 the mixing behavior can differ significantly across scales and using the simplification sequences we capture the behavior on all scales without recomputing the MS-complex. Domain scientists interact with a visualization of the segmented surface and select an appropriate persistence value based on their physical intuition of a correct segmentation of bubbles.

Application specifics. The techniques described so far are a direct application of Morse theory to automatically analyze scientific data. However, in some cases more application specific notions can be helpful. We apply two main adaptations: First, exclusive maxima or minima hierarchies, and second, oriented persistences.

The first case arises when one is only interested in either the maxima or the minima. For example, when segmenting and counting bubbles in the mixing layer the number and location of minima (spikes) is of no interest. In this case we restrict the simplification to only consider maximum-saddle pairs for cancellation. This saves some computation and disk-space but more importantly makes the simplification of maxima more flexible. Considering Figure 4, imagine $v$ being canceled with one of its neighboring minima. Once $v$ is canceled, $u$ and $w$ are no longer connected. As a result their corresponding bubbles cannot merge (at least not directly). By disallowing all minimum-saddle cancellation we ensure that the maxima/bubble simplification is as flexible as possible. The segmentation of interest is no longer the full MS-complex but only the complex of stable manifolds (called Morse complex). Because the Morse complex only changes at maximum-saddle configurations, canceling the maximum-saddle pair with smallest persistence still corresponds to the $L_{\infty}$-optimal simplification.

The concept of oriented persistences is a special adaptation to the problem of analyzing the mixing layer. The mixing layer often develops inverted maxima below the primary surface. They are called inverted since the surface normal and the function gradient (in the ambient $3 \mathrm{D}$ space) point in opposite directions. Such maxima clearly do not correspond to bubbles and therefore should not be counted. Inverted maxima are removed by artificially defining their persistence to be negative guaranteeing that they are removed before any other maximum. Since we never count critical points with persistence equal or below zero (such points represent topological noise) this effectively removes inverted maxima from all considerations without changing the pipeline. Inversions are detected during the isosurface extraction by labeling every vertex as either inverted (when light fluid is above heavy fluid) or non-inverted (heavy fluid above light fluid).

Temporal tracking. In addition to gathering statistics on the number of topological structures, understanding how these structures relate to each other is equally important. The case of a collection of $N$ bubbles growing in time is qualitatively different than the case of $N / 2$ bubbles dying and the remaining $N / 2$ bubbles splitting. Counting bubbles does not reveal this difference. We track bubbles over time using a method similar to Samtaney et al. [23]. 
Given a persistence threshold, we simplify the MS-complex of the envelope surface of each time step by repeated maximum-saddle cancellations. We view these cancellations as merging the two bubbles represented by the stable manifolds of the two maxima (the canceled maxima and its existing pair in the Morse complex). For example, in Figure 4, critical point $u$ merges with $w$ becoming its child. Given this parent-child information, a bubble is represented by the union of its stable manifold with those of its children. For each bubble we compute the centroids of its faces in the envelope surface and compute correspondences between centroids in adjacent time steps using Euclidean distance. We create a graph whose nodes are the parent maxima over all time steps. For each correspondence between face centroids we insert an edge between the parent maxima in the graph. Thus, the edges of the graph are weighted according to the number of corresponding face centroids. By selecting different criteria for merge/split events we can construct a family of graphs, one of which is shown in Figure 8.

Interaction. We can visualize the bubble structure of an envelope surface at a given persistence by coloring all triangles in the stable manifold representing a bubble with the same color. In order to interact with the MS-complex of an envelope surface at full resolution we store the parent-child information described in the previous paragraph in a separate file for each time step. This file contains one 16-bit integer for each triangle indicating the stable-manifold of lowest persistence to which it belongs, and a list of records, one for each stable manifold in the Morse complex. Each stable manifold record contains the position of its maximum, the persistence at which it is simplified, and the index of its parent in the hierarchy. For a given persistence threshold, the index of the stable manifold of each triangle is obtained by starting at its initial maximum and traversing that maximum's chain of parents until the threshold is reached. We modified the streaming mesh viewer of Isenburg et. al. [18] to read the parent-child information and simplify it along with an input mesh. The simplifier is grid-based, so that each cell in the grid ends up with a single vertex and only a subset of triangles survive in the simplified mesh. We simply keep the parent-child information of these simplified triangles so that each triangle can be colored appropriately at an interactively set persistence threshold.

\section{Results}

We present results for both Borg $\left(1152^{3}, 5.8 \mathrm{~GB}\right.$ per time step) and BGL $\left(3072^{3}, 9.1 \mathrm{~GB}-109 \mathrm{~GB}\right.$ per time step). Because of the extreme data sizes and limited available disk space we use every second time step in each simulation. The analysis was performed on 68 dualprocessor nodes of a Linux based visualization cluster. In most cases jobs were parallelized over time across the nodes of the cluster. The

\begin{tabular}{|l|cc|cc|}
\hline & \multicolumn{2}{|c|}{ Borg } & \multicolumn{2}{c|}{ BGL } \\
& $\mathrm{T}=0$ & $\mathrm{~T}=758$ & $\mathrm{~T}=0$ & $\mathrm{~T}=246$ \\
\hline Midplane Ext. & $37 \mathrm{~s} / 5 \mathrm{MB}$ & $37 \mathrm{~s} / 5 \mathrm{MB}$ & $127 \mathrm{~s} / 36 \mathrm{MB}$ & $127 \mathrm{~s} / 36 \mathrm{MB}$ \\
Midplane Seg. & $10 \mathrm{~s} / 361 \mathrm{~KB}$ & $10 \mathrm{~s} / 504 \mathrm{~KB}$ & $62 \mathrm{~s} / 180 \mathrm{~KB}$ & $66 \mathrm{~s} / 1.4 \mathrm{MB}$ \\
Isosurface Ext. & $14 \mathrm{~s} / 7.9 \mathrm{MB}$ & $27 \mathrm{~s} / 154 \mathrm{MB}$ & n.a. & n.a. \\
Isosurface Seg. & $2 \mathrm{~s} / 62 \mathrm{~KB}$ & $25 \mathrm{~s} / 144 \mathrm{~KB}$ & n.a. & n.a. \\
\hline
\end{tabular}

Table 1. Performance data for different stages of the pipeline shown in Figure 2. Execution times as well as resulting file sizes are shown for both the first and last time step of both datasets.

data was reblocked in a preprocess because of the large number of files written by the simulation code (1728 files/time-step for Borg and up to 65536 files/time-step for BGL). The reblocking time was highly variable, depending on file system load. In most cases a Borg time step required at least 20 minutes to reblock, while a BGL time step required at least 3 hours.

Table 1 shows the running times and resulting file sizes for extraction and segmentation of midplane and isosurfaces on a single node of the cluster. Each operation is shown on the first and last timestep of the corresponding dataset which is roughly equivalent to the fastest and slowest execution across the temporal range. The size of the isosurface refers to a standard ASCII obj file. All other formats are uncompressed binary. The midplanes are extracted from the raw simulation data, and the large number of files accessed caused longer run times. Once the envelope surfaces of all available time steps have been segmented, the combined statistics on all bubble counts at all time steps using any persistence is represented in a single uncompressed binary file of $14 \mathrm{Mb}$ for the Borg and $2 \mathrm{Mb}$ for BGL. Similar files are created for the density and $Z$ velocity information of Figures 5 and 6.

Figures 5 and 6 show critical point counts for $Z$ velocity maxima and density minima at the midplane versus time. The correlation between $Z$ velocity and density is used in the model presented in [7] to predict the late-time growth rate of the mixing layer. Qualitatively, the $Z$ velocity and density curves have similar features indicating a degree of correlation. The hierarchical approach based on persistence filtration of the MS complex introduces the ability to explore features at multiple scales. As the plots show, counting the strongest extrema appears to extract the expected phases in the mixing. These phases were described in [8] and evidence for phase III (the mixing transition) were observed in [7] in the probability density function of the fraction of the heavy fluid.

In Figure 5 the mixing transition (phase III) appears to be quite abbreviated compared to the mixing transition in the BGL plot (fig. 6 left). It is not clear if the actual onset of phase IV occurs much later in time and is not discernible from visual inspection of the plots. The asymptotic behavior, as illustrated by the curve fits, shows a slower rate of decrease in bubble counts (indicating a slowing in the increase in bubble size) in the Borg data in contrast to the BGL data. The asymptotic behavior of the large persistence curves does not appear to be tending to the $t^{-4}$ behavior expected from a purely dimensional analysis $[8,6]$. However, it may be necessary to run the BGL simulation to later time to discern whether the slope will continue decreasing. Furthermore, the presence of a subgrid-scale model for diffusivity used to create Borg may change the properties of the mixing transition since the diffusion and mixing of the two fluids dominates the entrainment rate in that regime.

For Borg, we also tracked bubbles, as defined by a Morse-Smale segmentation of the upper envelope (defined as the $98 \%$ density isosurface). The dataset was subsampled in $X$ and $Y$ by 2 and subsampled in $Z$ by 1 . In experiments with subsets of the data the subsampling rate did not appear to affect bubble counts except at very early times.

Figure 7 illustrates the bubble segmentation process. The images on the right side of the plot are all taken at the same time step for different persistences, showing that choosing the persistence threshold is important for getting an intuitively correct bubble segmentation. The images to the left and below are from the same curve, showing the increase in size of the bubbles, as indicated by the surface area of the set of Morse-Smale cells of all child maxima grouped with the parent maxima of each bubble.

The $2.39 \%$ persistence-threshold curve in Figure 7 shows three linear (power-law) regimes. Comparing this curve to the curve in Figure 5 shows that the pattern of slopes is inverted. The rate of decrease in the number of bubbles (which is correlated with increasing mixing width) is slow, then fast, then slow in Figure 7. In Figure 5 the rate of decrease in density minima is fast, slow, fast, which coincides with the phases observed in [7]. There is an intriguing tail-off on all three curves in Figure 7, although it is not clear that running the simulation to later time was practical given the grid resolution and the subgridscale numerical models used.

To develop a better understanding of the evolution of the bubbles we also tracked their birth,deaths, merges, and splits. Figure 8 shows results for a small part (1/27th) of the upper envelope surface of Borg at a persistence of $2 \%$. Using the maxima tracking discussed in Section 5 we compute a history of bubble births, deaths, merges, and splits and encode this information in a traditional merge-split graph. The rendered images are created by picking bubbles in the last time step and coloring them consistently through out the simulation by a simple majority-wins algorithm based on edge weights. As expected, the merge-split graph is dominated by merges rather than deaths and contains very few splits. This suggests that bubbles at the late stage mixing are created by the merging of smaller bubbles instead of the independent growth of a single bubble. However, visual examination of movies of the mixing layer indicates that bubble stagnation may 


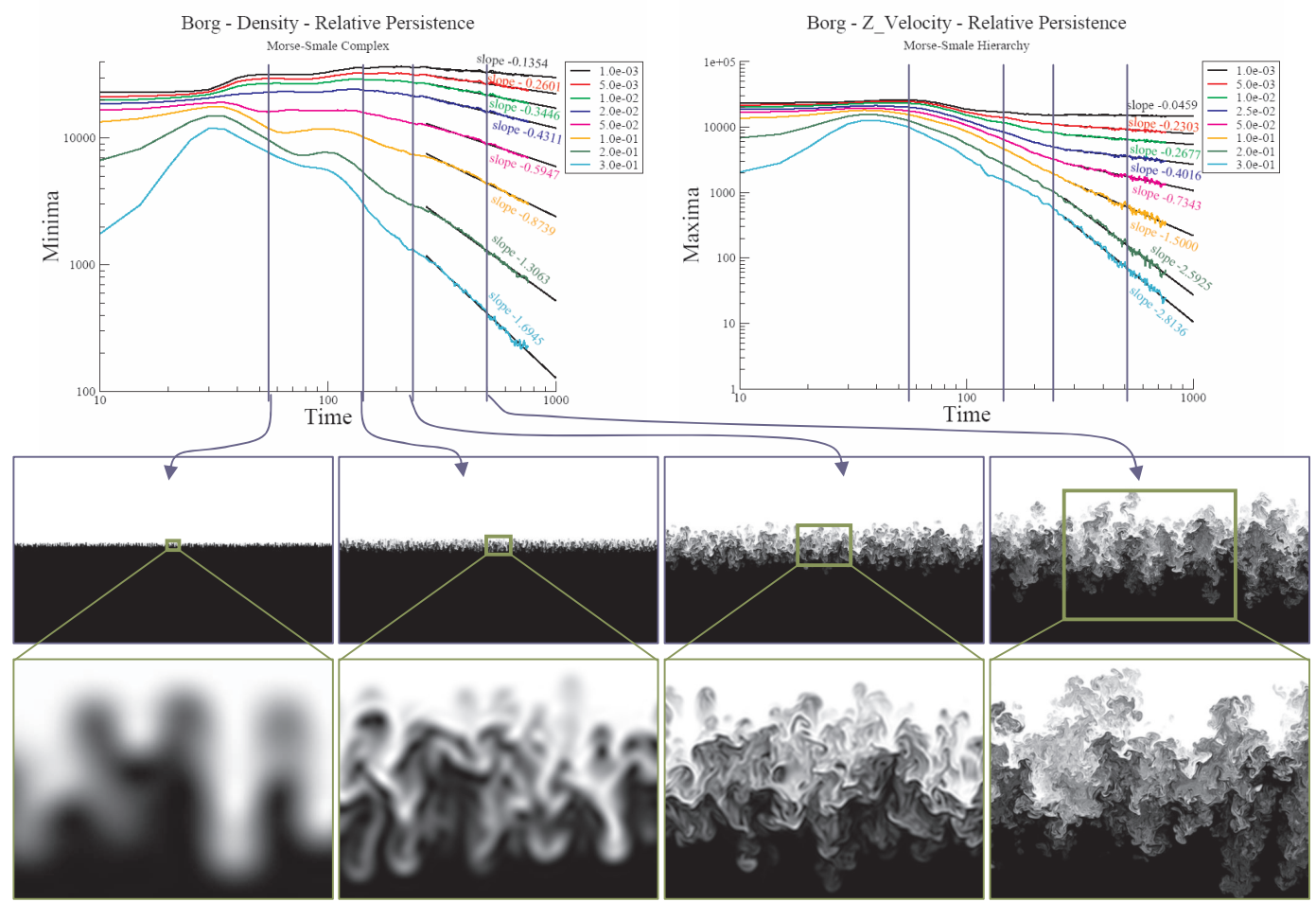

Fig. 5. Critical point counts for the $1152^{3}$ Borg simulation, showing density minima (left) and $Z$ velocity maxima (right) at the midplane versus time. Density minima indicate the locations where light fluid is intersecting the midplane. At left, the four phases of the mixing are suggested by four lines at distinct times in the middle of each phase and arrows indicating the corresponding $X Z$ slices of the density field, where white denotes the heavy fluid. The linear regions of the high persistence curves suggest power-law behavior. Recall that reductions in the number of high persistence critical points is correlated with the structures in the flow becoming larger. Thus, larger structures are indicative of growth of the mixing region. The asymptotic behavior is fitted for late time.

also play an important role. Some death events may require additional information (such as the $Z$ location or velocity) to disambiguate them from merge events.

\section{Conclusion}

In this paper we have demonstrated how topological segmentation based on Morse theory can be used to analyze the properties of mixing flow. We have presented a novel definition of bubbles in the mixing layer as stable manifolds of maxima of the height function. Furthermore, we have developed a suite of efficient algorithms forming a complete pipeline to perform multi-scale analysis of large and complex datasets. Except for the tracking, all algorithms are combinatorial making them stable even under extreme circumstances. It should be noted that the techniques described in this paper are in no-way limited to fluid dynamics research. On the contrary, the tools described here can be applied to any domain in which features can be indicated by the critical points of a function defined on a manifold.

We have extracted counts of critical points that correlate with growth of the mixing layer and the associated growth in the size (and therefore reduction in number) of features in the flow. Our results match previously observed phases of the mixing process which suggests that our topological approach is valid. However, there are differences in the power-law behavior of the bubble counts derived from the envelope surfaces and the density minima counts on the midplanes. It is not clear at this time what accounts for these differences. We believe a better tracking system might shed more light on the late time steps when bubbles become complicated multi-scale structures.

Our geometrically based temporal tracking approach achieves the expected qualitative results. By corresponding every face in the surface, a simple majority-win algorithm reduces mis-identification of events, except some death events which require additional information. We expect that the merge-split graph will be an important tool in the analysis of mixing behavior.
The results presented here highlight the need to develop more sophisticated metrics which do not require a qualitative approach to selecting a persistence threshold. It is clear that the flow features of interest are multi-scale, but it is not clear how to combine the different scales into a fully quantitative metric.

There exist some real limitations to attempting to track 3-D structures like bubbles using 2-D envelope surfaces. As the midplane images in Figure 6 show, the internal structure of bubbles at late time is complex. It appears that a robust and scalable implementation of 3-D Morse-Smale complexes is required to capture all relevant information in the mixing process. Finally, temporal-aware topological algorithms are an area of future research when analyzing time-varying problems.

\section{Acknowledgements}

The authors thank William Cabot and Andrew Cook for the datasets. This work was performed under the auspices of the US Department of Energy by University of California Lawrence Livermore National Laboratory under contract No. W-7405-Eng-48. UCRL-JRNL-222696.

\section{References}

[1] U. Alon and D. Shvarts. Two-phase flow model for rayleigh-taylor and richtmeyer-meshkov mixing. In Proc. Fifth Int. Workshop on Compressible Turb. Mixing. World Scientific, 1996.

[2] C. Bajaj B. Sohn. Time-varying contour topology. IEEE Trans. on Vis. and Comp. Graph., 12(1):14-25, 2005.

[3] P.-T. Bremer, H. Edelsbrunner, B. Hamann, and V. Pascucci. A topological hierarchy for functions on triangulated surfaces. IEEE Trans. on Vis. and Comp. Graph., 10(4):385-396, 2004.

[4] P.-T. Bremer and V. Pascucci. A practical approach to two-dimensional scalar topology. H. Hauser, H. Theisel, and H. Hagen, editors, To appear. 2006.

[5] H. Carr, J. Snoeyink, and M. van de Panne. Simplifying flexible isosurfaces using local geometric measures. In IEEE Vis., 2004. 

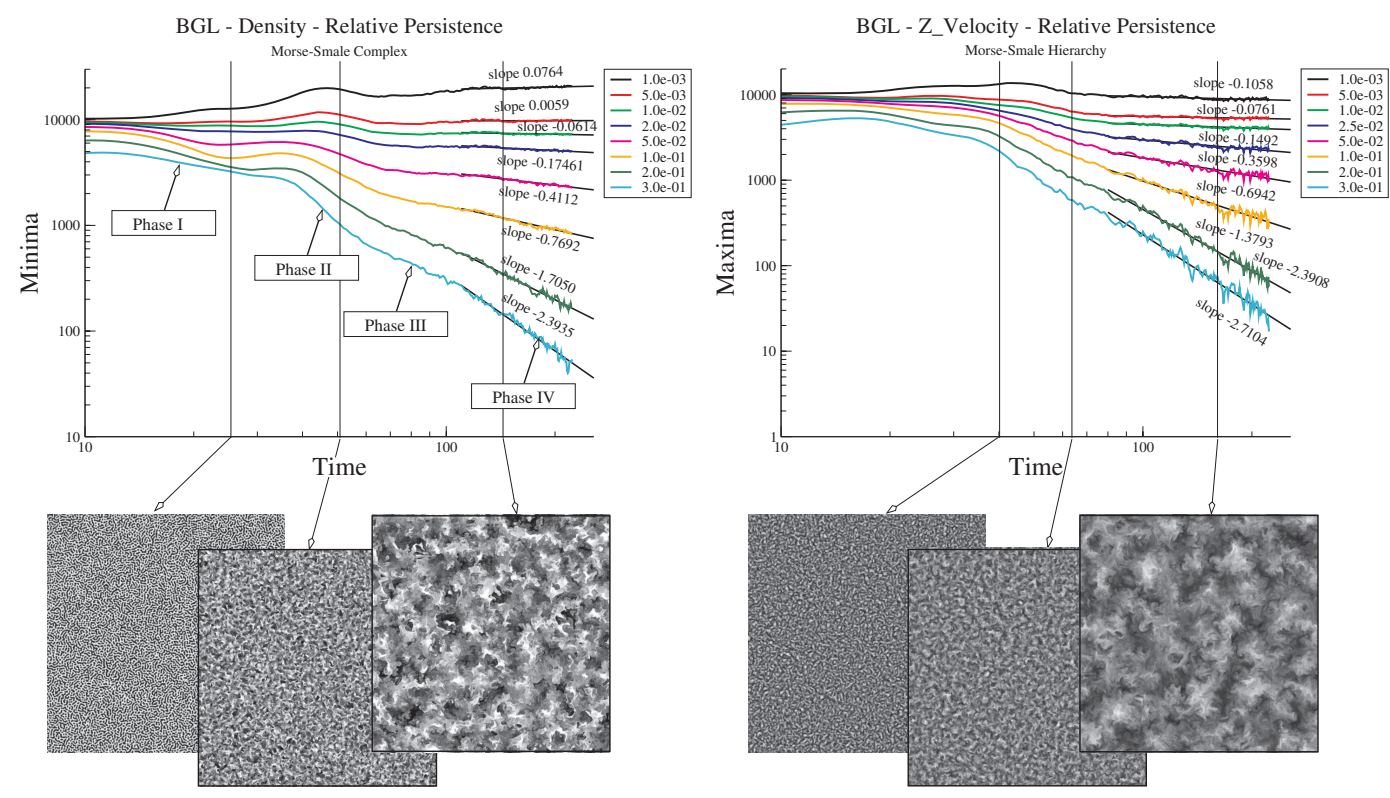

Fig. 6. Critical-point counts for several persistence values on the midplane of BGL $\left(3072^{3}\right)$. (Left) Number of minima of the density field vs. time. The four phases of the mixing process are suggested by the changes in slope of the curve as indicated by the arrows. In particular, a power law behavior (slope on the log-log plot) is suggested from phase II (coupled growth), phase III (mixing transition with decreased growth rate) and phase IV (strong turbulence and an asymptotic growth rate). Below the plot are gray scale images of the density field at the midplane, showing from left to right phase I decoupled growth, phase II weak turbulence with a small amount of mixing (gray regions) and late time strong turbulence with large areas of mixed fluid indicated by gray. The asymptotic behavior at late time is estimated with curve fits. (Right) Number of maxima of the $Z$-velocity at the midplane at several persistence thresholds vs. time. The maxima segment regions of movement of light fluid upwards into the heavy fluid. A transition between phase II and III is suggested, but a clear transition to phase IV is not apparent. Below the plot are images of the $Z$ component of the velocity field on the midplane.

[6] T.T. Clark. A numerical study of the statistics of a two dimensional Rayleigh-Taylor mixing layer. Phys. Fluids, 15:2413-2423.

[7] A.W. Cook, W. Cabot, and P.L. Miller. The mixing transition in RayleighTaylor instability. J. Fluid Mech., to appear, 2004.

[8] A.W. Cook and P. E. Dimotakis. Transition stages of Rayleigh-Taylor instability between miscible fluids. J. Fluid Mech., 443:66-99.

[9] D. Kartoon, D. Oron, L. Arazi, and D. Shvarts. Three-dimensional multimode rayleigh-taylor and richtmeyer instabilities at all density ratios. Laser and Particle Beams, 21:327-334.

[10] W. de Leeuw and R. van Liere. Collapsing flow topology using area metrics. In Proc. IEEE Vis., pages 349-354. 1999.

[11] H. Edelsbrunner and J. Harer. Jacobi sets of multiple Morse functions. In Foundations of Computational Mathematics, Minneapolis 2002, pages 37-57. Cambridge Univ. Press, England.

[12] H. Edelsbrunner, J. Harer, A. Mascarenhas, and V. Pascucci. Timevarying Reeb graphs for continuous space-time data. In 20th Symp. on Comp. Geom., pages 366-372, New York, NY, USA, 2004.

[13] H. Edelsbrunner, J. Harer, V. Natarajan, and V. Pascucci. Morse-Smale complexes for piecewise linear 3-manifolds. In Proc. 19th Sympos. Comput. Geom., pages 361-370, 2003.

[14] H. Edelsbrunner, J. Harer, and A. Zomorodian. Hierarchical MorseSmale complexes for piecewise linear 2-manifolds. Discrete Comput. Geom., 30:87-107, 2003.

[15] C. Garth, X. Tricoche, and G. Scheuermann. Tracking of vector field singularities in unstructured 3D time-dependent datasets. In Proc. IEEE Visualization, pages 329-336. 2004.

[16] A. Gyulassy, V. Natarajan, V. Pascucci, P.-T. Bremer, and B. Hamann. Topology-based simplification for feature extraction from 3D scalar fields. IEEE Trans. on Vis. and Comp. Graph., 2006. to appear.

[17] J. L. Helman and L. Hesselink. Visualizing vector field topology in fluid flows. IEEE Comp. Graph. and Appl., 11(3):36-46. 1991.

[18] M. Isenburg, P. Lindstrom, S. Gumhold, and J. Snoeyink. Large mesh simplification using processing sequences. In Proc. IEEE Vis., pages 465-472. 2003

[19] G. Ji and H.-W. Shen. Efficient isosurface tracking using precomputed correspondence table. In Proc. Symp. on Vis., pages 283-292. 2004.

[20] G. Ji, H.-W. Shen, and R. Wegner. Volume tracking using higher dimensional isocontouring. In Proc. IEEE Vis., pages 209-216. 2003.
[21] J. Milnor. Morse Theory. Princeton Univ. Press, New Jersey, 1963.

[22] F. Reinders, F. H. Post, and H. J. W. Spoelder. Visualization of timedependent data with feature tracking and event detection. The Visual Computer, 17(1):55-71, 2001.

[23] R. Samtaney, D. Silver, N. Zabusky, and J. Cao. Visualizing features and tracking their evolution. IEEE Computer, 27(7):20-27, 1994.

[24] G. Scheuermann and X. Tricoche. Topological methods in flow visualization. In Visualization Handbook, pages 341-356. Elsevier, 2004.

[25] D. Silver and X. Wang. Tracking and visualizing turbulent 3D features. IEEE Trans. on Vis. and Comp. Graph., 3(2):129-141, 1997.

[26] D. Silver and X. Wang. Tracking scalar features in unstructured datasets. In Proc. IEEE Vis., pages 79-86. 1998.

[27] A. Szymczak. Subdomain-aware contour trees and contour tree evolution in time-dependent scalar fields. In Proc. Shape Modeling International (SMI) '05, pages 136-144. IEEE Computer Society, 2005.

[28] H. Theisel, T. Weinkauf, H.-C. Hege, and H.-P.Seidel. Saddle connectors - An approach to visualizing the topological skeleton of complex 3D vector fields. In Proc. IEEE Vis., pages 225-232, 2003.

[29] H. Theisel, T. Weinkauf, H.-C. Hege, and H.-P. Seidel. Topological methods for $2 \mathrm{~d}$ time-dependent vector fields based on stream lines and path lines. IEEE Trans. on Vis. and Comp. Graph., 2005.

[30] X. Tricoche, G. Scheuermann, and H. Hagen. A topology simplification method for 2d vector fields. In IEEE Vis., pages 359-366. 2000.

[31] X. Tricoche, T. Wischgoll, G. Scheuermann, and H. Hagen. Topology tracking for the visualization of time-dependent two-dimensional flows. Computer \& Graphics, 26(2):249-257, 2002.

[32] G. Weber, G. Scheuermann, H. Hagen, and B. Hamann. Exploring scalar fields using critical isovalues. In M. Gross, K. I. Joy, and R. J. Moorhead, editors, Proc. IEEE Vis., pages 171-178. 2002.

[33] T. Weinkauf, H. Theisel, K. Shi, H.-C. Hege, and H.-P.Seidel. Extracting higher order critical points and topological simplification of 3D vector fields. In Proc. IEEE Vis., pages 559-566. 2005. 


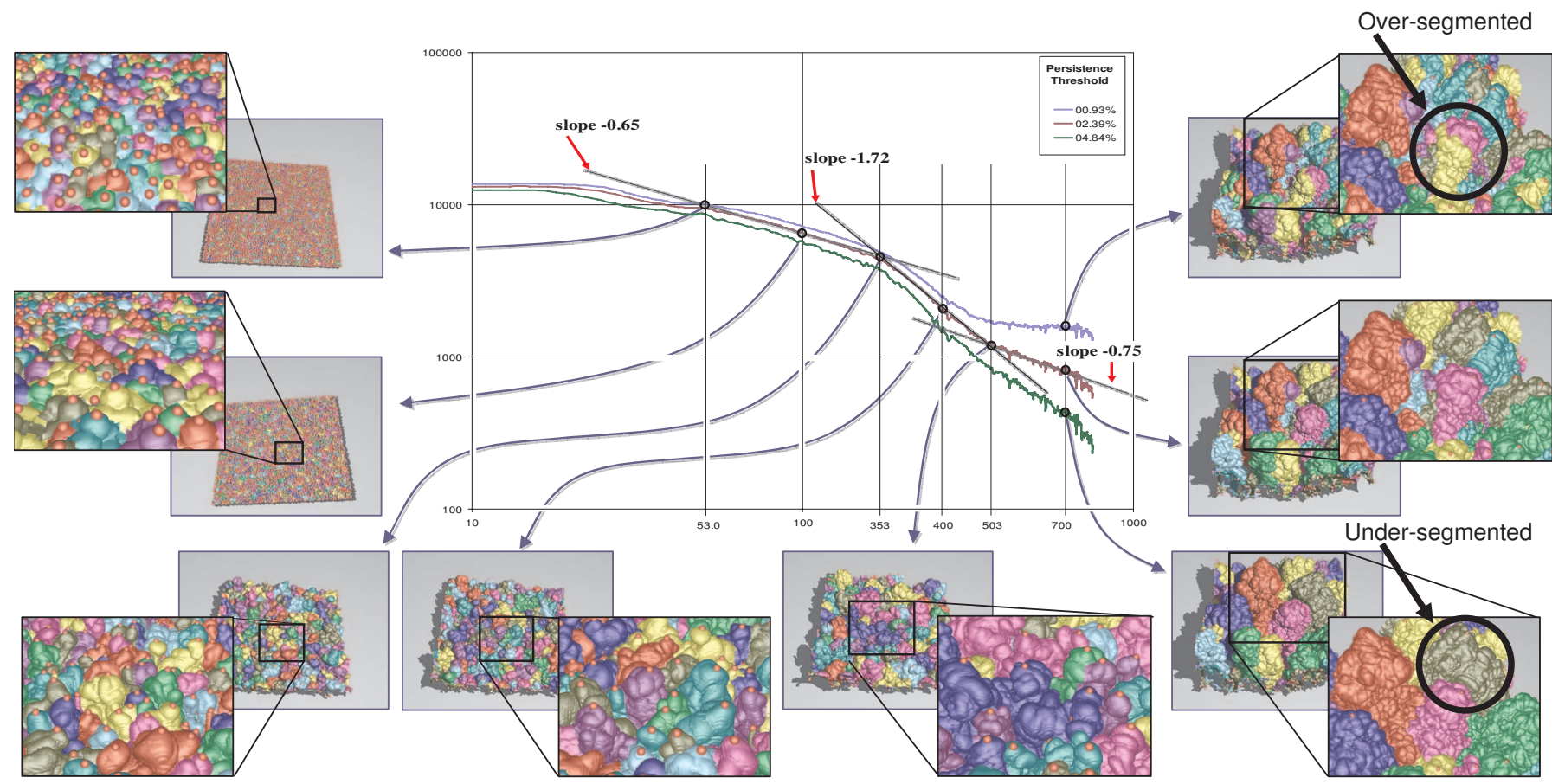

Fig. 7. The plot depicts bubble counts of the envelope surface for three persistence values. Three regions of power-law behavior are shown by the curve fits in gray to the $2.39 \%$ persistence curve. To the right of the plot, the MS-segmentation at three persistence values for time 700 . To the left and below the plot, the bubble segmentation along the $2.39 \%$ persistence curve at various times. Each maximum along with the Morse cells of its child-maxima are colored the same.

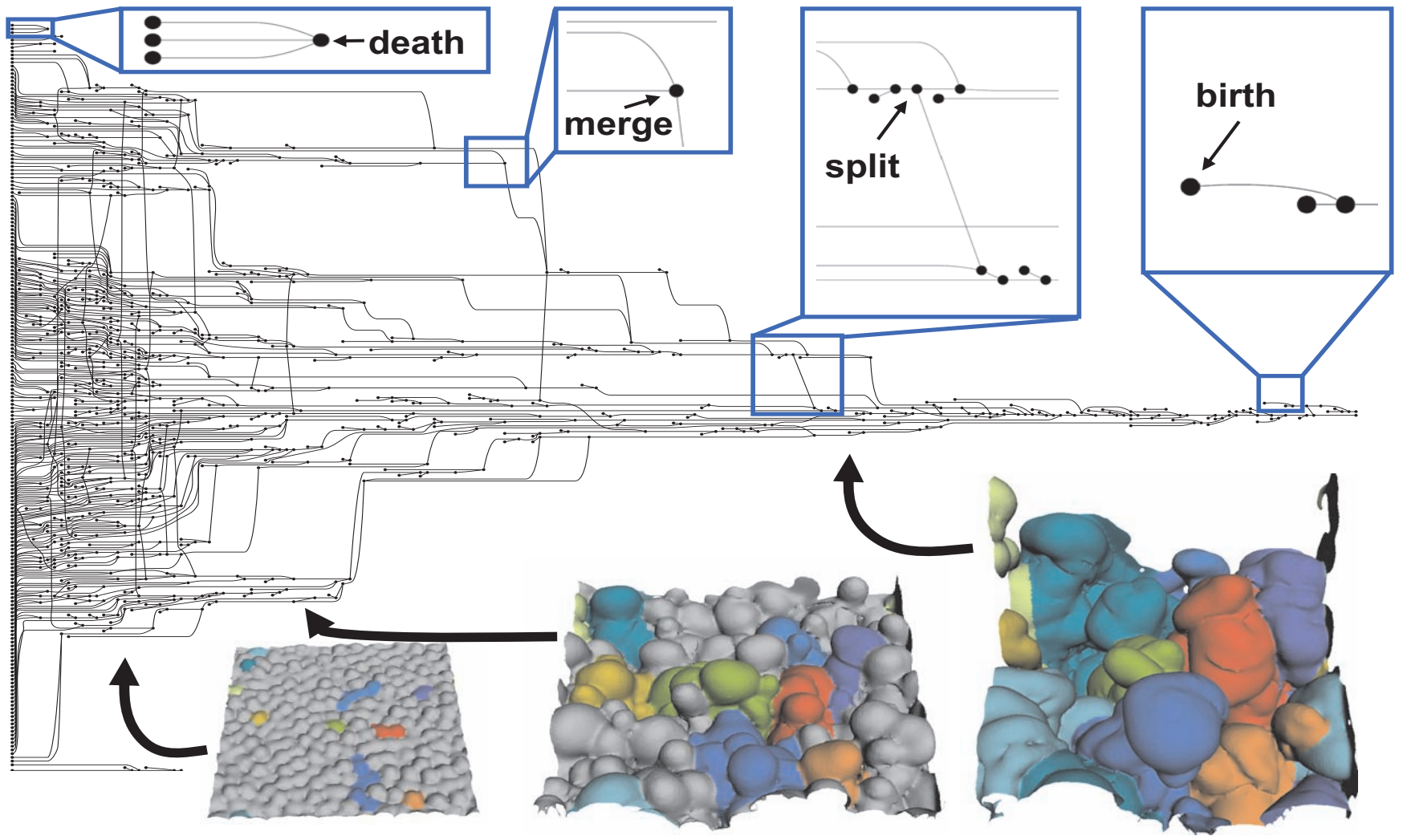

Fig. 8. A subset of the merge-split graph of bubbles over time at a persistence of $2 \%$. Edges with normalized weight less than $40 \%$ are discarded. The three images at bottom show ten bubbles colored consistently at three time steps indicated by the curved arrows. The graph is dominated by merges, which implies either that late stage bubbles are created by the joining of smaller bubbles or that smaller bubbles stagnate and are subsumed into the larger bubbles. 